\title{
Standards der Fissuren- und Grübchenversiegelung
}

Roswitha Heinrich-Weltzien, Jan Kühnisch

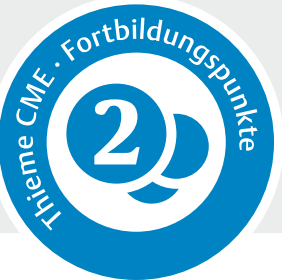

\begin{abstract}
Karies ist weltweit eine der häufigsten chronischen Erkrankungen in allen Lebensphasen. In den Industrienationen mit der breiten Verfügbarkeit fluoridhaltiger Zahnpasten ist der Kariesbefall auch bei Nichtrisikopatienten im Kindes- und Jugendalter auf die Fissuren und Grübchen der bleibenden Molaren konzentriert. Die Versiegelung dieser Kariesprädilektionsstellen ist eine zahnflächenspezifische Präventionsmaßnahme zur Vorbeugung einer Fissuren- und Grübchenkaries. Basierend auf der überarbeiteten S3-Leitlinie „Fissuren- und Grübchenversiegelung“ werden die aktuellen Empfehlungen zum klinischen Prozedere zusammenfassend dargestellt.
\end{abstract}

\section{Hintergrund}

Die Versiegelung von Fissuren und Grübchen wird seit etwa 5 Dekaden zur Prävention und Kontrolle kariöser Läsionen von Milch- und bleibenden Zähnen genutzt [1]. Trotz ihrer kariespräventiven Wirksamkeit wird sie noch immer zu wenig zur Vorbeugung einer Kariesinitiation und -progression der Fissuren- und Grübchenkaries in der täglichen Praxis genutzt [2]. In der Bundesrepublik Deutschland ist die Fissuren- und Grübchenversiegelung (F-G-V) der bleibenden Molaren seit 1993 eine abrechenbare kassenärztliche Leistung (IP5) für gesetzlich versicherte Patienten im Alter von 5 bis 18 Jahren. Wie epidemiologische Studien der letzten 20 Jahre zeigen, waren durchschnittlich 2 bis 3 Molaren pro Kind versiegelt, wobei ein Großteil der F-G-V nur partiell intakt waren [3]. Weiterhin haben Kinder und Jugendliche aus sozial benachteiligten Gruppen, mit einem Migrationshintergrund und mit Behinderungen wesentlich seltener und weniger F-G-V $[4,5]$.

Seit 2005 steht der Zahnärzteschaft in Deutschland die S3-Leitlinie „Fissuren- und Grübchenversiegelung“ zur Verfügung, die nunmehr in der 2. aktualisierten Form vorliegt [3].

\section{Fissuren- und Grübchenkaries}

Ursachen der erhöhten Kariesanfälligkeit von Fissuren und Grübchen der bleibenden Molaren sind das zerklüftete Fissuren- und Grübchenrelief sowie die geringe Schmelzdicke am Fissurenfundus [3]. Der Zahndurchbruch erstreckt sich über einen längeren Zeitraum, der in der Regel mit einer ungenügenden Reinigung der Okklusalflächen durch die kindlichen Patienten verbunden ist. Darüber hinaus kann der Biofilm aus dem Fissurenrelief mit der Zahnbürste nur begrenzt entfernt werden ( $\vee$ Abb. 1 a und b). Aufgrund dieser Risikofaktoren treten häufig initial kariöse Läsionen unmittelbar nach dem Durchbruch der Molaren auf ( $\bullet$ Abb. $2 \mathbf{a}$ und $\mathbf{b}$ ). Neben den anatomischen und biologischen Besonderheiten spielt auch das Gesundheitsverhalten des Patienten wie die frequente Aufnahme zuckerhaltiger Nahrungsmittel und/oder Getränke, die Häufigkeit und Qualität der täglichen Mundhygiene und die Fluoridbilanz eine wesentliche Rolle bei der Initiation des kariösen Prozesses [6].

\section{Fissuren- und Grübchenversiegelung}

Der präventive Verschluss der kariesanfälligen Fissuren und Grübchen zur Vorbeugung einer Kariesinitiation und/oder der Arretierung kariöser Frühstadien mit einem dünnfließenden Versieglermaterial wird als F-G-V definiert [7]. Es ist eine zahnflächenspezifische Präventionsmaßnahme, die präventive Effekte an anderen Zahnflächen ausschließt. Neben der F-G-V sollten eine zahngesunde Ernährung und adäquate häusliche Mundhygienemaßnahmen sowie eine indikationsgerechte häusliche und professionelle Fluoridapplikation Bestandteile einer umfassenden präventiven Betreuung sein [3].

Zielgruppe für die F-G-V sind vorrangig Kinder und Jugendliche, da sie von dieser Präventionsmaßnahme unmittelbar nach dem Durchbruch der bleibenden Molaren am meisten profitieren. Die Empfehlung gilt für Kinder und Jugendliche mit und ohne Komorbiditäten [3]. 


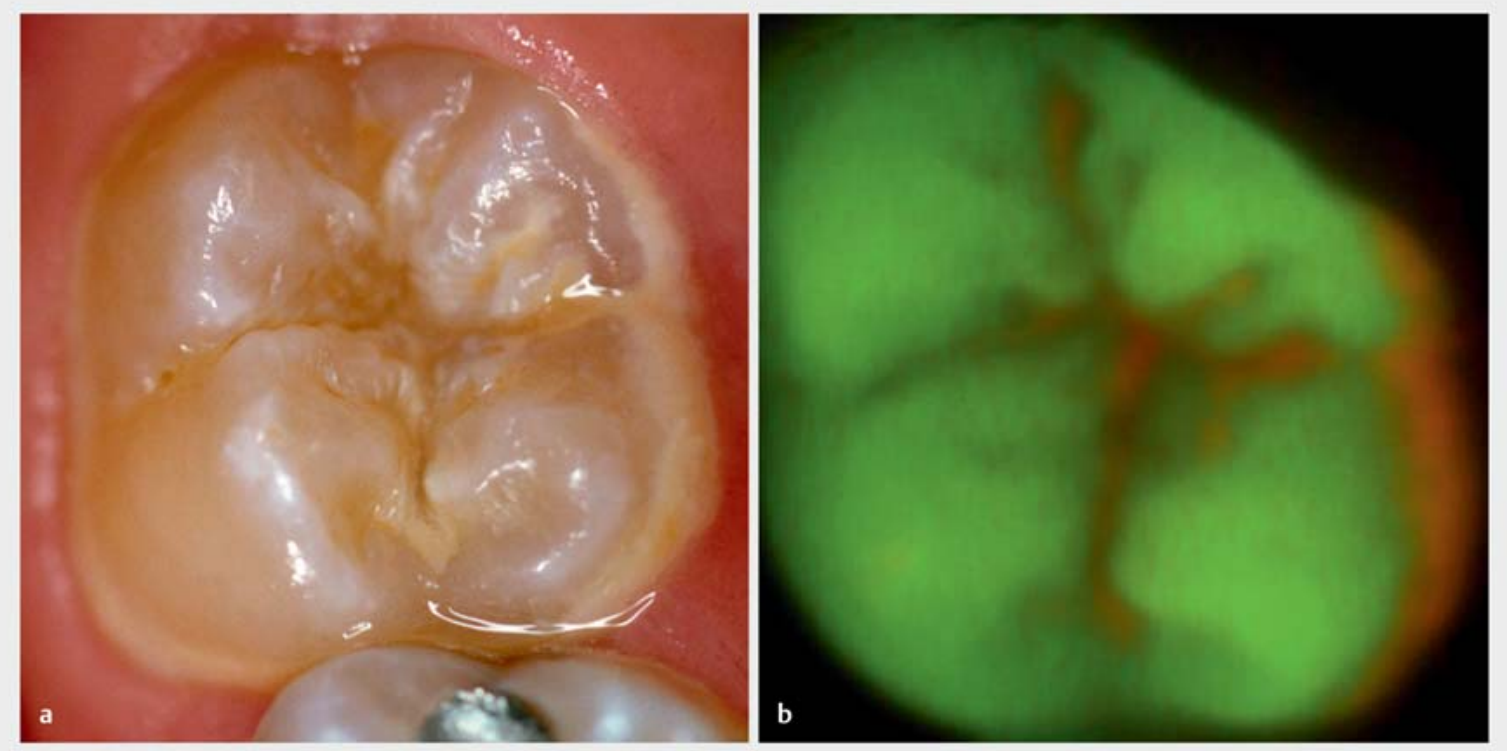

- Abb. 1 Okklusale initial kariöse Läsion an Zahn 47 und ausgeprägte zirkuläre marginale Plaqueablagerungen (a). Die quantitative lichtinduzierte Fluoreszenzaufnahme des Zahnes 47 (b) visualisiert die Demineralisation der okklusalen Fissur sowie die Plaqueakkumulation in der Fissur und marginal (Rotfluoreszenz).
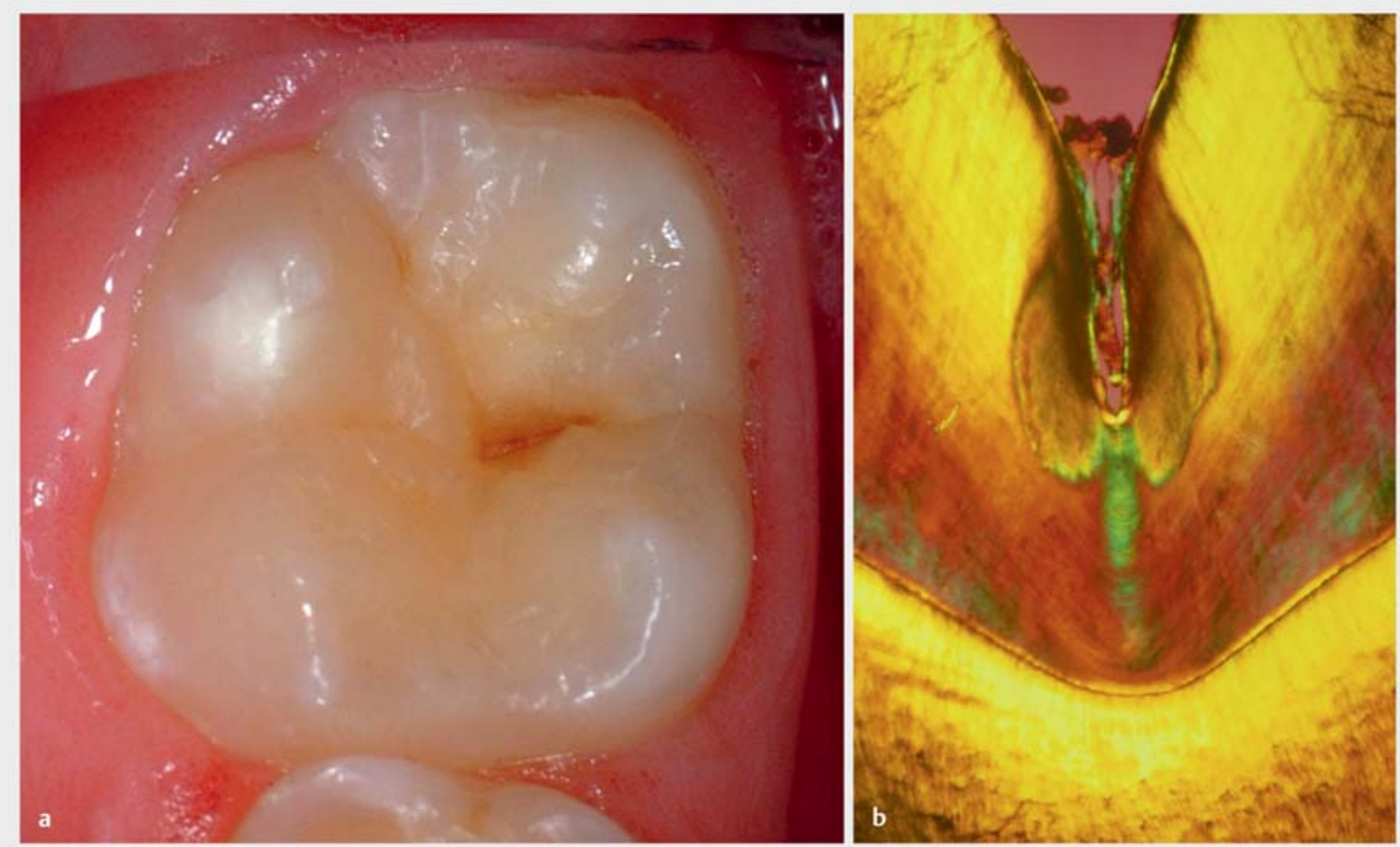

- Abb. 2 Okklusale Mikrokavitation an Zahn 46 eines 7-jährigen Patienten (a). Polarisationsoptisches Bild einer nicht kavitierten initial kariösen Läsion am Fissurenfundus (b). 

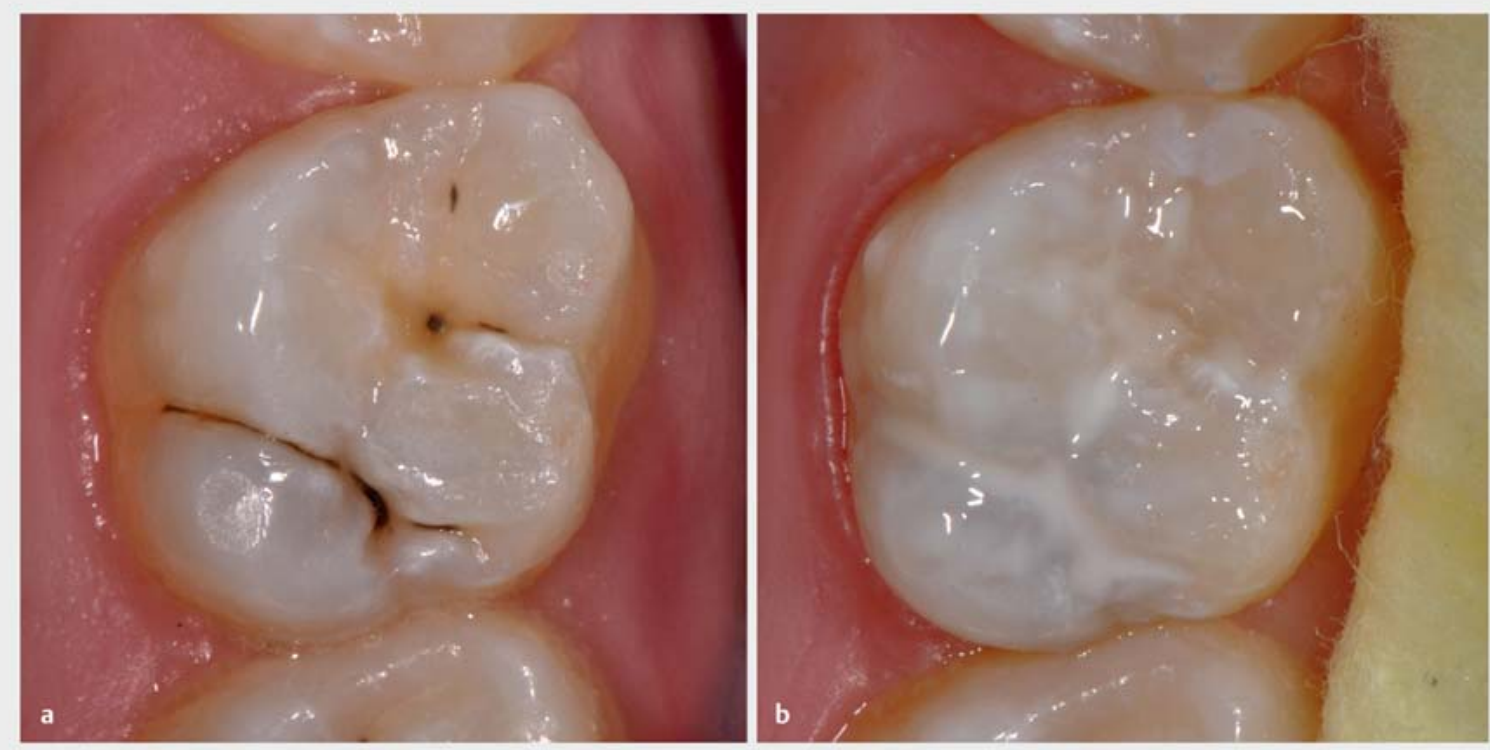

- Abb. 3 Zahn 16 mit einer nicht kavitierten kariösen Läsion vor (a) und nach (b) der Applikation einer Fissuren- und Grübchenversiegelung.

\section{Diagnostik vor der Fissuren- und Grübchenversiegelung}

Vor der F-G-V ist grundsätzlich eine sorgfältige kariesdiagnostische Untersuchung indiziert (Anschauliche Grafik zum diagnostischen Entscheidungsprozess zur Fissuren- und Grübchenversiegelung in der Kurzversion der Leitlinie auf Seite 5. Zu erreichen unter https://eref. thieme.de/MKEMW). Dabei wurde als Schlüsselempfehlung formuliert, dass der visuellen Diagnostik [8] an der gereinigten und getrockneten Okklusalfläche der Vorzug eingeräumt werden soll und beim Vorliegen nicht kavitierter kariöser Läsionen ergänzende diagnostische Verfahren, wie die Bissflügelröntgenaufnahme oder lichtoptische Untersuchung (z. B. DIAGNOdent) zur Detektion versteckter Dentinläsionen (Kontraindikation für $\mathrm{F}-\mathrm{G}-\mathrm{V}$ ) indiziert sind [9].

Nach der kariesdiagnostischen Untersuchung wäre aus praktischer Sicht eine der folgenden Diagnosen zu stellen:

- gesunde, kariesfreie Fissur bzw. Grübchen

- Fissur bzw. Grübchen mit einer nicht kavitierten kariösen Läsion (Synonyme: Initialkaries, präkavitierte Schmelzkaries, beginnende oder frühe Karies, Hidden oder versteckte Karies, Kariesvorstufe)

- Kavitation bzw. Dentinkaries im Bereich der Fissuren bzw. Grübchen

Ergänzend zur Kariesdiagnostik werden die Einschätzung des allgemeinen Kariesrisikos bzw. der Kariesaktivität kariöser Läsionen sowie der prioritäre Einsatz der F-G-V bei
Kindern und Jugendlichen mit einem erhöhten Kariesrisiko und bestehender Kariesaktivität als Schlüsselempfehlung gegeben.

\section{Merke}

Die klinische Diagnostik eines erhöhten Kariesrisikos erfolgt durch die Detektion und Diagnostik kariöser Läsionen.

\section{Kariesrisiko}

Ein guter Prädiktor für eine zukünftige kariöse Entwicklung sind neben dem aktuellen Kariesbefall klinisch einfach zu erfassende nicht kavitierte kariöse Läsionen [10]. Aus klinischer Sicht werden die ersten 5 Jahre nach dem Zahndurchbruch als Zeitraum eines erhöhten Kariesrisikos des jeweiligen Zahnes angesehen [10].

\section{Kariesaktivität}

Kennzeichen der Aktivität kariöser Läsionen ist das Vorhandensein von Plaque (Biofilm) auf ihrer Oberfläche, ein weißlich rauer Zahnschmelz sowie die Präsenz von erweichtem Dentin bei kavitierten Läsionen [6]. Die Kariesaktivität wird maßgeblich durch das allgemeine Kariesrisiko und Alter des Patienten beeinflusst [9].

\section{Indikationen zur Fissuren- und Grübchenversiegelung}

In folgenden klinischen Situationen ist die Fissuren- und Grübchenversiegelung der bleibenden Molaren indiziert: 
- Kariesfreie Fissuren und Grübchen bei Patienten mit einem erhöhtem Kariesrisiko. Dazu zählen u. a. Patienten mit Karieserfahrung im Milchgebiss und Patienten, die bereits einen kariösen bleibenden Molaren aufweisen.

- kariesfreie Fissuren und Grübchen mit einem kariesanfälligen Fissurenrelief unabhängig von der Kariesrisiko-Einschätzung

- Fissuren und Grübchen mit nicht kavitierten kariösen Läsionen unabhängig von der Kariesrisiko-Einschätzung ( $\triangleright$ Abb. $\mathbf{3}$ a und $\mathbf{b}$ )

- Fissuren und Grübchen an hypomineralisierten oder hypoplastischen Zähnen unabhängig von der Kariesrisiko-Einschätzung

- Fissuren und Grübchen bei Patienten mit Allgemeinerkrankungen bzw. körperlichen und/oder geistigen Behinderungen, die eine effektive tägliche Mundhygiene nur begrenzt umsetzen können

- partiell oder vollständig verloren gegangene Fissurenversiegelungen sollten bei unverändertem Kariesrisiko repariert bzw. erneuert werden ( $\bullet$ Abb. 4 )

- An Milchmolaren und anderen bleibenden Zähnen kann bei einem erhöhten individuellen oder zahnflächenspezifischen Risiko die F-G-V erwogen werden ( $\triangleright$ Abb. 5 a und b).

\section{Relative Kontraindikationen zur}

\section{Fissuren- und Grübchenversiegelung}

- Ist der betreffende Zahn noch nicht vollständig in die Mundhöhle durchgebrochen und sind die Okklusalfläche bzw. palatinalen/bukkalen Grübchen nicht oder

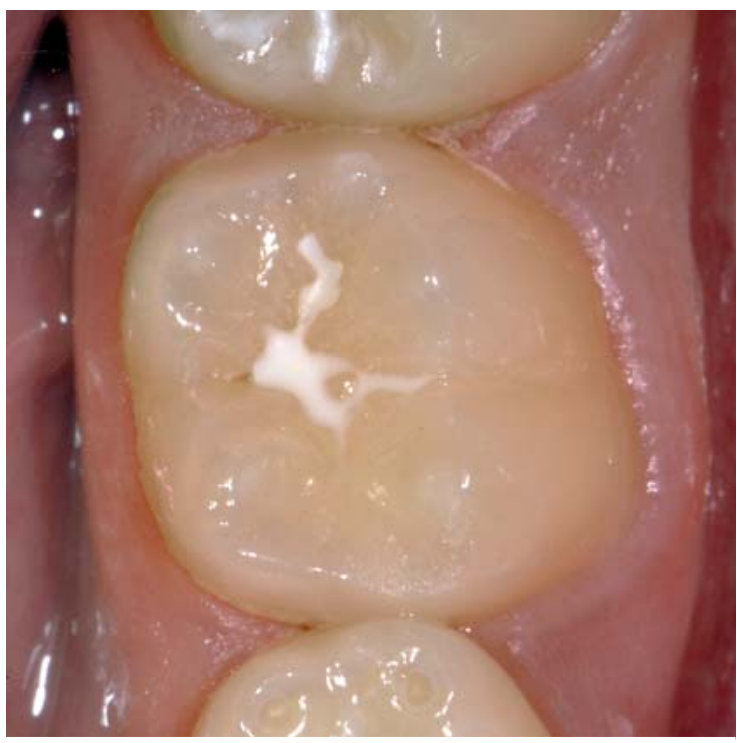

- Abb. 4 Zahn 36 mit Verlust der Fissurenversiegelung in peripheren Fissurenbereichen und Mikrokavitation im exponierten zentralen Fissurenareal. Die linguale Plaqueablagerung und Gingivitis signalisiert ein hohes Kariesrisiko des Patienten, sodass die Nachversiegelung indiziert ist.

nur begrenzt einer adäquaten Trockenlegung zugänglich, ist auf die Versiegelung vorerst zu verzichten. Bis zum vollständigen Zahndurchbruch haben eine adäquate Plaqueentfernung und die Lokalapplikation von Fluorid(lack)en Vorrang. Bei Kariesrisiko-Patienten kann die temporäre F-G-V mit einem Glasionomerzement (GIZ; Prä-F-V) erwogen werden. Es ist eine einfache, präventive, aber provisorische Interimslösung.
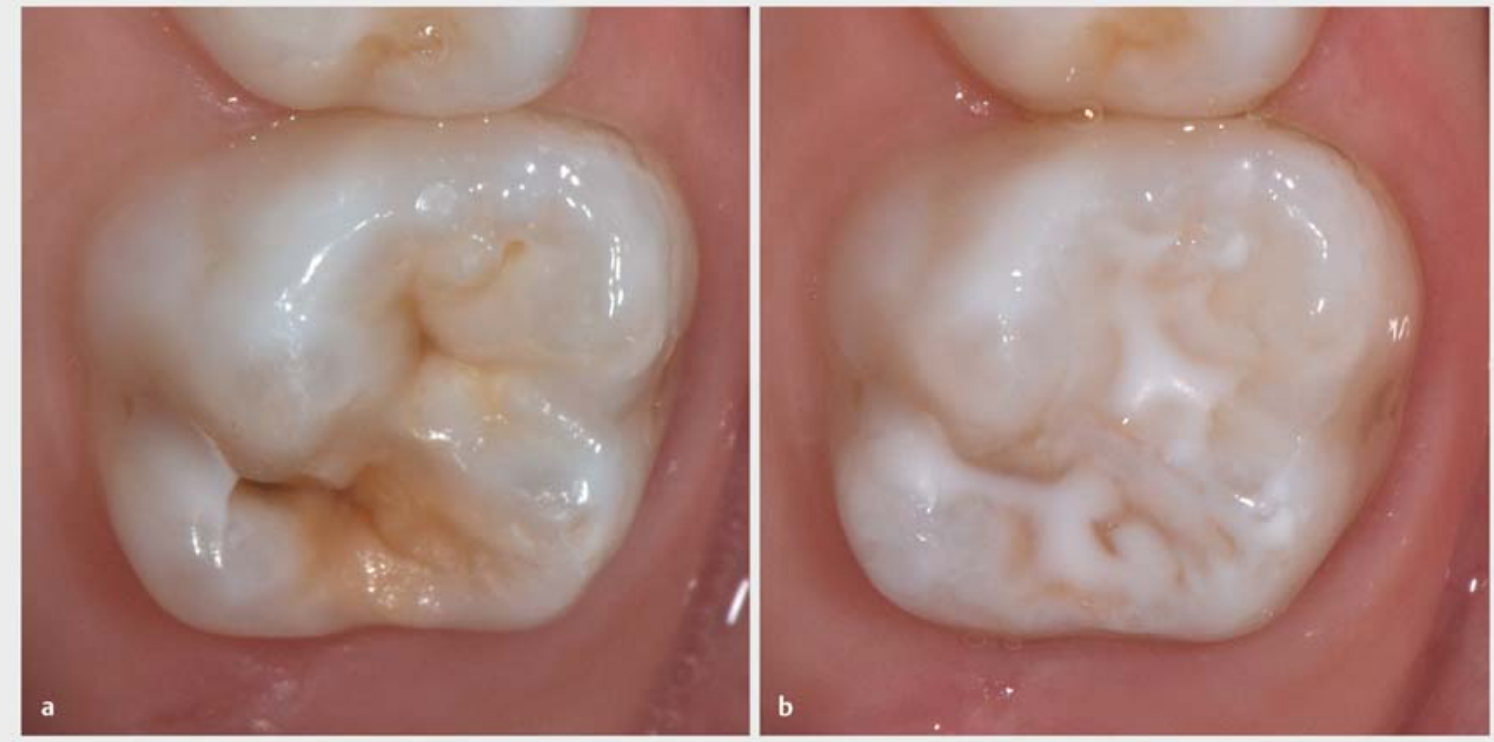

- Abb. 5 Okklusale Hypomineralisation (deciduous molar hypomineralisation) an Zahn 65, die mit einem erhöhten Kariesrisiko verbunden ist, vor (a) und nach der Fissurenversiegelung (b). 
- Bei Zähnen mit einer Dentinkaries im Bereich der Fissuren bzw. Grübchen ist die Versiegelung kontraindiziert und die minimalinvasive Füllungstherapie angezeigt.

- Unmittelbar vor der Exfoliation stehende Milchzähne bedürfen keiner Versiegelung.

\section{Merke}

Eine absolute Kontraindikation zur F-G-V besteht bei einer nachgewiesenen Allergie gegenüber Versiegelungsmaterialien oder einzelnen Materialbestandteilen.

\section{SCHLÜSSELEMPFEHLUNG}

Als Schlüsselempfehlungen wurde in der Leitlinie zur Indikation der F-G-V konsertiert:

- Die F-G-V soll indikationsgerecht an gesunden Fissuren zur Kariesprävention und an Fissuren mit nicht kavitierten kariösen Läsionen zur Kariesarretierung zum Einsatz kommen. Empfehlungsstärke: stark/Konsensstärke: stark.

- Während bei Nichtrisikopatienten die Indikation zur F-G-V an kariesfreien Zähnen restriktiv gestellt werden soll, profitieren Kariesrisiko-Patienten von der F-G-V, weshalb die Versiegelung bei diesen Patienten bevorzugt durchgeführt werden soll. Empfehlungsstärke: stark/Konsensstärke: stark.

- An Fissuren und Grübchen mit nicht kavitierten kariösen Läsionen soll die Indikation zur Versiegelung im Kindes- und Jugendalter unabhängig von der Kariesrisiko-Einschätzung mit dem Ziel der Kariesarretierung gestellt werden. Empfehlungsstärke: stark/Konsensstärke: stark.

\section{Klinisches Vorgehen bei der Fissuren- und Grübchenversiegelung}

\section{Reinigung der Zahnoberfläche}

Die Zahnreinigung mit rotierenden Bürstchen mit/ohne Verwendung einer Prophylaxepaste ist ein Routinevorgehen. Sie ist einfach, schnell und kindgerecht durchführbar und wurde in zahlreichen klinischen Studien eingesetzt.

Die fehlende „Tiefenreinigung“ enger Fissuren wurde bei Verwendung von Prophylaxe- bzw. Polierbürsten früher als Nachteil betrachtet, der durch den Einsatz von Pulver-Wasser- bzw. Partikelstrahlgeräten kompensiert werden sollte. Während In-vitro-Untersuchungen nach zusätzlicher Pulver-(Wasser-)Strahlreinigung zu teilweise verbesserten Verbundwerten, einer reduzierten Microleakage oder einer besseren Reinigung führten [11, 12], zeigten klinische Studien keine Unterschiede zwischen einzelnen Formen der Zahnreinigung nach 1 Jahr Beobachtungszeit $[13,14]$.

\section{SCHLÜSSELEMPFEHLUNG}

Die Zahnreinigung ist ein unverzichtbarer Teilarbeitsschritt der F-G-V und soll daher immer vor der Versiegelung erfolgen. Der Arbeitsschritt ist Grundlage für eine korrekte kariesdiagnostische Untersuchung an den Fissuren und Grübchen. Empfehlungsstärke: stark/Konsensstärke: stark.

\section{Trockenlegung}

Für die Langlebigkeit der F-G-V ist eine gute Trockenlegung wesentlich. Vergleichende Untersuchungen zur Verwendung der absoluten (Kofferdam) und relativen Trockenlegung (Watterollen) wiesen jedoch keine signifikanten Unterschiede der Überlebenszeit von F-G-V nach $[15,16]$. Bei der relativen Trockenlegung mit Watterollen ist jedoch unabdingbar, dass die Vier-Hand-Technik praktiziert wird.

\section{SCHLÜSSELEMPFEHLUNG}

Eine sichere Trockenlegung soll bei der F-G-V die Einhaltung der relevanten Arbeitsschritte, Konditionierung, Materialauftrag und Polymerisation, gewährleisten. Kann kein vierhändiges Arbeiten mit relativer Trockenlegung im Praxisalltag umgesetzt werden, wird die Applikation der F-G-V unter Zuhilfenahme von Kofferdam empfohlen. Empfehlungsstärke: Stark/Konsensstärke: Stark.

\section{Konditionierung der Schmelzschicht}

Die Säurekonditionierung ist das Standardvorgehen zur Herstellung eines adhäsiven Verbundes zwischen Zahnschmelz und methacrylatbasierten (Versiegelungs-)Kunststoffen. Sie ist seit Jahrzehnten Garant für die Langlebigkeit von adhäsiv befestigten Restaurationen oder F-G-V. Mit der Entfernung der aprismatischen Schmelzschicht wird durch die Exposition der Schmelzprismen ein mikroretentives Oberflächenrelief erzielt, dass eine enge Verzahnung mit dem hydrophoben Versiegelungskunststoff erlaubt. 
Die Schmelzkonditionierung wird in der Regel mit 35- bis $37 \%$ iger Ortho-Phosphorsäure in Gelform durchgeführt. Im Schrifttum dominieren klinische Studien, die eine Ätzzeit von 60 oder 30-40 Sekunden verwandten; nur wenige Arbeitsgruppen konditionierten den Zahnschmelz vor der F-G-V kürzer [3]. Nach gründlichem Absprayen der Säure und forcierter Trocknung muss eine kreidig-weiße Schmelzoberfläche sichtbar sein; sie ist das Charakteristikum des erfolgreichen Ätzvorgangs.

\section{SCHLÜSSELEMPFEHLUNGEN}

1. Die Säurekonditionierung stellt das Vorgehen der Wahl zur Konditionierung des Zahnschmelzes vor der F-G-V dar. Daher soll dieser Arbeitsschritt zur Anwendung kommen. Empfehlungsstärke: stark/ Konsensstärke: stark.

2. Die Einwirkzeit der Säure soll am unbehandelten Zahnschmelz vor der konventionellen F-G-V mindestens 30 Sekunden betragen. Ein opakes Ätzmuster gilt als adäquates Ergebnis des Ätzvorgangs. Empfehlungsstärke: stark/Konsensstärke: stark.

3. Eine Verkürzung der Säurekonditionierung auf weniger als 30 Sekunden kam in einigen klinischen Studien zum Einsatz. Die Ergebnisse zeigen ein heterogenes Retentionsverhalten mit zum Teil sehr niedrigen Raten intakter Versiegelungen nach 2 Jahren Liegedauer. Es fehlen aussagekräftige und langfristige klinische Studien zu der Fragestellung, auf welche Zeit die Säurekonditionierung verkürzt werden kann, ohne dass mit Retentionseinbußen zu rechnen ist. Empfehlungsstärke: offen/Konsensstärke: stark.

4. Die Anwendung von selbstkonditionierenden Adhäsiven stellt eine Möglichkeit dar, den klinischen Arbeitsprozess zu verkürzen. Allerdings erreichen die bislang dokumentierten Retentionsraten nicht die mit dem konventionellen Vorgehen publizierten Überlebensraten. Daher kann die klinische Anwendung selbstkonditionierender Adhäsive gegenwärtig nicht vorbehaltlos empfohlen werden. Empfehlungsstärke: offen/Konsensstärke: stark.

\section{Materialien zur Fissuren- und Grübchenversiegelung}

Die Eignung von Materialien zur F-G-V wird anhand des Retentionsverhaltens in Bezug zur Liegedauer beurteilt. Im Rahmen der Leitlinie [3] wurde dazu eine Metaanalyse von klinischen Studien mit einer Mindestlaufzeit von 2 Jahren durchgeführt. Im Ergebnis dessen wurde gezeigt, dass das Retentionsverhalten von F-G-V materialabhängig ist. Das günstigste Retentionsverhalten wiesen auto- und lichtpolymerisierende Versieglermaterialien auf. Nach 4 Jahren Liegezeit waren etwa $80 \%$ der Lichtpolymerisate bei Anwendung der Säurekonditionierung intakt; bei Verwendung selbstkonditionierender Haftvermittler reduzierte sich der Anteil intakter F-G-V auf unter $50 \%$ bereits nach 2 Jahren Liegedauer [15]. Vergleichsuntersuchungen zwischen auto- und lichtpolymerisierenden Materialien ermittelten keine signifikanten Unterschiede in den Retentionsraten [16]. Ein unzureichendes Retentionsverhalten wiesen Kompomere bei ihrer Verwendung zur F-G-V auf, was durch die fehlende Säurekonditionierung bedingt sein dürfte [15]. Der Einsatz fließfähiger Komposite wird aufgrund ihrer höheren Viskosität vor allem bei der minimalinvasiven Füllungstherapie (Synonym: erweiterte F-G-V) gesehen. GIZ hatten im Vergleich mit allen anderen Versieglermaterialien die niedrigsten Rententionsraten; nach 2 Jahren waren 80$90 \%$ aller F-G-V nicht mehr intakt bzw. verloren gegangen.

\section{SCHLÜSSELEMPFEHLUNG}

Die Schlüsselempfehlung zu den Materialgruppen lautet:

1. Es sollen Materialgruppen mit einer hohen Retentionsrate und damit Überlebenswahrscheinlichkeit bevorzugt in der klinischen Praxis eingesetzt werden. Dazu zählen niedrigvisköse methacrylatbasierte Versiegelungskunststoffe, die in Verbindung mit Säurekonditionierung angewendet werden.

2. Bei Zähnen im Durchbruch bzw. wenn keine adäquate Trockenlegung möglich ist, kann alternativ der Einsatz von GIZ erwogen werden. Empfehlungsstärke: stark/Konsensstärke: stark.

3. Lichtpolymerisate sollten als Ein-KomponentenMaterialien im Vergleich zu Autopolymerisaten bevorzugt verwendet werden. Die Materialien sind weniger techniksensitiv zu verarbeiten, da der Anmischvorgang entfällt und die sofortige Lichtpolymerisation die Behandlungszeit verkürzt. Empfehlungsstärke: moderat/Konsensstärke: stark. 


\section{SCHLÜSSELEMPFEHLUNG}

Für die Arbeitsschritte der Applikation, Polymerisation, Okklusionskontrolle und Politur der F-G-V wurden folgende Schlüsselempfehlungen ausgesprochen [3]: 1. Die Applikation des Versiegelungsmaterials soll grazil im Fissurenrelief erfolgen. Materialüberschüsse, die zu okklusalen Vorkontakten und einem partiellen oder vollständigen Retentionsverlust führen können, sollen vermieden werden. Empfehlungsstärke: stark/Konsensstärke: stark.

2. Die Polymerisationszeit ist abhängig von der Lichtintensität und dem Versiegelungsmaterial und soll in der Regel 20 Sekunden betragen (Beachten: alle Versiegelungsanteile müssen vom Licht ausreichend erfasst werden). Nach der Aushärtung soll eine Okklusionskontrolle erfolgen; interferierende Überschüsse sollen korrigiert werden. Empfehlungsstärke: stark/Konsensstärke: stark.

3. Zum Entfernen der oberflächlichen Sauerstoffinhibitionsschicht soll eine Politur der Fissuren- und Grübchenversiegelung erfolgen. Zur Remineralisation geätzter, aber nicht versiegelter Schmelzareale wird die Lokalapplikation eines Fluoridpräparats empfohlen. Empfehlungsstärke: stark/Konsensstärke: stark.

\section{Monitoring der Fissuren- und Grübchenversiegelung}

F-G-V sollen aufgrund eines möglichen Retentionsverlustes alle 6 bis 12 Monate kontrolliert werden $[17,18]$. Bei Patienten mit einem hohen Kariesrisiko sollten die Kontrollintervalle 12 Monate nicht überschreiten [17].

\section{Merke}

Bei einem partiellen oder vollständigen Retentionsverlust soll die Nachversiegelung anhand der Indikationsempfehlungen geprüft werden. Verbliebenes Versiegelungsmaterial muss hinsichtlich seiner Retention kontrolliert werden; die Entfernung fest anhaftender Materialreste ist nicht erforderlich.

\section{Schlussfolgerungen}

Die F-G-V sollte wesentlicher Bestandteil eines präventiven zahnärztlichen Betreuungskonzepts von Kindern und Jugendlichen sein. Sie ist eine sichere und effektive Maßnahme, deren kariespräventiver Nutzen in systematischen Übersichtsarbeiten der Cochrane Collaboration belegt wurde $[19,20]$. Während die F-G-V an gesunden Fissuren und Grübchen die Kariesinitiation vorbeugt, stoppt sie die Progression von nicht kavitierten (initial kariösen) Läsionen [1]. Dafür ist die vollständige und intakte F-G-V conditio sine qua non. Die vorliegende aktualisierte Leit- linie dient dem Zahnarzt als Handlungsempfehlung für die Durchführung der einzelnen Arbeitsschritte der F-G-V im Sinne eines Qualitätsmanagements [3].

\section{KERNAUSSAGEN}

- Die Fissuren- und Grübchenversiegelung (F-G-V) ist eine zahnflächenspezifische Präventionsmaßnahme.

- Der kariespräventive Nutzen wurde für die bleibenden Molaren in Metaanalysen und systematischen Übersichtsarbeiten als hoch eingeschätzt.

- Die F-G-V ist an gesunden Fissuren zur Kariesprävention und an Fissuren mit nicht kavitierten kariösen Läsionen zur Kariesarretierung indiziert.

- Niedrigvisköse methacrylatbasierte Versiegelungskunststoffe sind mit der Säurekonditionierung anzuwenden, dabei sind Lichtpolymerisate als EinKomponenten-Materialien den Autopolymerisaten vorzuziehen.

\section{Hinweis}

Die Lang-, Kurz- und Patientenfassung der S3-Leitlinie „Fissuren- und Grübchenversiegelung“ (AWMF Registernummer 083-002) sowie der Leitlinienreport sind von den Webseiten der AWMF und der DGZMK abrufbar.

\section{Autorinnen/Autoren}

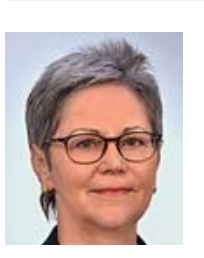

\section{Roswitha Heinrich-Weltzien}

Prof. Dr. med. dent. Jahrgang 1950. 19691974 Studium der Zahnheilkunde an der Friedrich-Schiller-Universität Jena. 19741979 Fachzahnarzt-Ausbildung Kinderzahnheilkunde an der Poliklinik für Präventive Zahnheilkunde und Kinderzahnheilkunde an der Medizinischen Akademie Erfurt. Seit 2009 Komm. Direktorin der Poliklinik für Präventive Zahnheilkunde und Kinderzahnheilkunde am Universitätsklinikum Jena. Schwerpunkte: Betreuung von Patienten mit chronischen Erkrankungen und Behinderungen, Orale Epidemiologie, Kariesdiagnostik und -prävention, Klinik der Kinder- und Jugendzahnmedizin

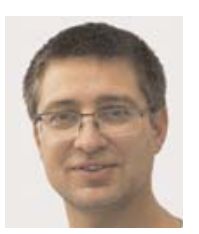

\section{Jan Kühnisch}

Prof. Dr. med. dent. Jahrgang 1971. 19911996 Studium der Zahnmedizin an der Universität Leipzig und Friedrich-Schiller-Universität Jena/Bereich Erfurt, 1998 Wrigley-Prophylaxe-Preis, 1999 Vivadent-Forschungspreis, 2002 Wrigley-Prophylaxe-Preis, 2003

Spezialisierung im Fachbereich „Kinder- und Jugendzahnheilkunde“, Oral-B blend-a-med Prophylaxe Preis, 2008/09 Habilitation und Ernennung zum Privatdozenten, 2015 Ernennung zum apl. Professor. Schwerpunkte: Kariologie - Epidemiologie, Prävention und Therapie, Strukturstörungen der Zähne, Traumatologie, Klinik der Kinder- und Jugendzahnmedizin 


\section{Interessenkonflikt}

Als korrespondierender Autor erkläre ich, dass mein Koautor mir mitteilte, in den letzten 3 Jahren keine wirtschaftlichen oder persönlichen Verbindungen im oben genannten Sinne gehabt zu haben. Ich selbst hatte ebenfalls keine derartigen Verbindungen in den letzten 3 Jahren.

\section{Korrespondenzadresse}

\section{Prof. Dr. Roswitha Heinrich-Weltzien}

Poliklinik für Präventive Zahnheilkunde und Kinderzahnheilkunde, Universitätsklinikum Jena Bachstraße 18

07743 Jena

roswitha.heinrich-weltzien@med.uni-jena.de

\section{Wissenschaftlich verantwortlich} gemäß Zertifizierungsbestimmungen

Wissenschaftlich verantwortlich gemäß Zertifizierungsbestimmungen für diesen Beitrag ist Prof. Dr. R. HeinrichWeltzien.

\section{Literatur}

[1] Wright JT, Crall J], Fontana M et al. Evidence-based Clinical Practice Guideline for the Use of Pit- and-Fissure Sealants. American Academy of Pediatric Dentistry, American Dental Association. Pediatr Dent 2016; 38: E120-E136

[2] Tellez M, Gray SL, Gray S et al. Sealants and dental caries: dentists' perspectives on evidence-based recommendations. J Am Dent Assoc 2011; 142: 1033-1040

[3] [Anonym]. S3-Leitlinie zur Fissuren- und Grübchenversiegelung, Langversion 2016, AWMF Registernummer: 083/002. Im Internet: http://www.awmf.org/leitlinien/detail/I/083002.html; Stand: 25.06 .2018

[4] Heinrich-Weltzien R, Walther M, Goddon I et al. Zahngesundheit erster Molaren bei westfälischen Migranten und deutschen Schülern. Bundesgesundheitsblatt 2014; 57: 128-134

[5] Hempel E, Limberger K, Möller $\mathrm{M}$ et al. Mundgesundheit von Erfurter Schüler/innen mit und ohne Behinderungen. Gesundheitswesen 2015; 77: 263-268

[6] Carvalho JC. Caries process on occlusal surfaces: Evolving evidence and understanding. Caries Res 2014; 48: 339-346

[7] Welbury R, Raadal M, Lygidakis NA. EAPD guidelines for the use of pit and fissure sealants. Eur J Paediatr Dent 2004, 5: 179-184
[8] Gimenez T, Piovesan C, Braga MM et al. Visual inspection for caries detection: A systematic review and metaanalysis. J Dent Res 2015; 94: 895-904

[9] Ismail Al, Tellez M, Pitts NB et al. Caries management pathways preserve dental tissues and promote oral health. Community Dent Oral Epidemiol 2013; 41: e12-e40

[10] Mejare I, Axelsson A, Dalen G et al. Caries risk assessment. A systematic review. Acta Odontol Scand 2014; 72: 81-91

[11] Garcia-Godoy F, Medlock JW. An SEM study of the effects of air-polishing on fissure surfaces. Quintessence Int 1988; 19: 465-467

[12] Sol E, Espasa E, Bol JR et al. Effect of different prophylaxis methods on sealant adhesion. J Clin Pediatr Dent 2000; 24: 211-214

[13] Donnan MF, Ball IA. A double-blind clinical trial to determine the importance of pumice prophylaxis on fissure sealant retention. Br Dent J 1988; 165:283-286

[14] Gillcrist JA, Vaughan MP, Plumlee GN et al. Clinical sealant retention following two different tooth-cleaning techniques. J Public Health Dent 1998; 58: 254-256

[15] Kühnisch J, Heinrich-Weltzien R, Kessler A et al. Evidenzbasierte klinische Empfehlungen zur Fissuren- und Grübchenversiegelung. Quintessenz 2017; 68: 1137-1146

[16] Muller-Bolla M, Lupi-Pegurier L, Tardieu C et al. Retention of resin-based pit and fissure sealants: A systematic review. Community Dent Oral Epidemiol 2006; 34: 321-336

[17] Irish Oral Health Services Guideline Initiative. Pit and fissure sealants: Evidence-based guidance on the use of sealants for the prevention and management of pit and fissure caries. 2010. Im Internet: www.ucc.ie/en/media/research/ohsrc/PitandFissureSealantsFull.pdf; Stand: 25.06.2018

[18] Smallridge J. UK National Clinical Guidelines in Paediatric Dentistry: Use of fissure sealants including management of the stained fissure in first permanent molars. Int J Paed Dent 2010; 20 (Suppl. 1): 3. doi:10.1111/j.1365-263X.2010.01086.x

[19] Ahovuo-Saloranta A, Hiiri A, Nordblad A et al. Pit and fissure sealants for preventing dental decay in the permanent teeth of children and adolescents. Cochrane Database Syst Rev 2008; (4): CD001830

[20] Ahovuo-Saloranta A, Forss H, Walsh T et al. Sealants for preventing dental decay in the permanent teeth. Cochrane Database Syst Rev 2013; (3): CD001830

\section{Bibliografie}

DOI https://doi.org/10.1055/a-0715-3590

ZWR - Das Deutsche Zahnärzteblatt 2018; 127: 468-477

(c) Georg Thieme Verlag KG Stuttgart · New York ISSN 0044-166X 


\section{Punkte sammeln auf CME.thieme.de}

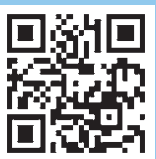

Diese Fortbildungseinheit ist 12 Monate online für die Teilnahme verfügbar.

Sollten Sie Fragen zur Online-Teilnahme haben, finden Sie unter cme.thieme.de/hilfe

eine ausführliche Anleitung. Wir wünschen viel Erfolg beim Beantworten

der Fragen!

Unter eref.thieme.de/CXBM29T oder über den QR-Code kommen Sie direkt zum Artikel zur Eingabe der Antworten.

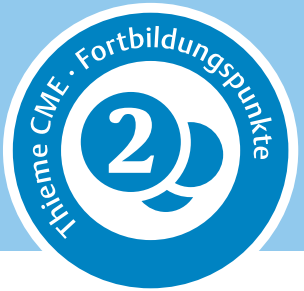

\section{Frage 1}

Was ist die Fissurenversiegelung?
A praxisrelevante Maßnahme zur Kariesvorbeugung
B zahnflächenspezifische Präventionsmaßnahme
C praktikable Therapiemaßnahme
D kostengünstige Präventionsmaßnahme
E Behandlungsmaßnahme für alle Kinder und Jugendliche

\section{Frage 2}

Wann ist die Fissuren- und Grübchenversiegelung indiziert?

A Wenn eine okklusale Mikrokavitation mit und ohne Dentinkaries vorliegt.

B Wenn das Okklusalrelief erster Molaren der Selbstreinigung zugängig ist.

C Wenn ein gesundes Fissurenrelief oder eine nicht kavitierte kariöse Läsion in der Fissur vorliegt.

D Wenn das Kariesrisiko des Patienten niedrig ist.

E Wenn der Patient älter als 18 Jahre ist.

\section{Frage 3}

Wann besteht eine absolute Kontraindikation zur Fissuren- und Grübchenversiegelung?

A bei einer Asthmaerkrankung des Patienten

$B$ bei einer nachgewiesenen Jodallergie des Patienten

$C$ bei einer nachgewiesenen Allergie gegenüber einzelnen Materialbestandteilen von Versiegelungsmaterialien

D bei Patienten mit Immunsuppression

E bei unvollständig durchgebrochenen ersten Molaren

\section{Frage 4}

In welcher der folgenden klinischen Situationen an Milchmolaren ist eine Fissuren- und Grübchenversiegelung in Erwägung zu ziehen?

A bei einer Hypomineralisationsstörung der Okklusalfläche und palatialen/bukkalen Grübchen

$\mathrm{B}$ bei einem unkooperativen Patienten

$C$ bei Patienten jünger als 6 Jahren

D bei kurz vor Exfoliation stehenden Milchmolaren

$\mathrm{E}$ bei allen Patienten mit Allgemeinerkrankungen

\section{Frage 5}

Welche der folgenden Aussagen zur Durchführung einer Fissuren- und Grübchenversiegelung trifft zu?

A Die Fissuren- und Grübchenversiegelung sollte immer unter Kofferdam erfolgen.

B Eine sichere Trockenlegung soll die Einhaltung der relevanten Arbeitsschritte - Konditionierung, Materialauftrag und Polymerisation - bei der Fissuren- und Grübchenversiegelung gewährleisten.

C Die Fissuren- und Grübchenversiegelung muss immer vierhändig durchgeführt werden.

D Die Fissuren- und Grübchenversiegelung sollte nach einer „Tiefenreinigung“ von engen Fissuren vorgenommen werden.

E Zur „Tiefenreinigung“ enger Fissuren sollten Pulver-Wasserbzw. Partikelstrahlgeräte zum Einsatz kommen.

\section{Frage 6}

Die Säurekonditionierung des unbehandelten Zahnschmelzes ist das Vorgehen der Wahl vor der konventionellen Fissurenversiegelung. Wie lang ist die Einwirkzeit der Säure?
A mindestens 120 Sekunden
B mindestens 60 Sekunden
C mindestens 30 Sekunden
D 30 bis 60 Sekunden
E 20 Sekunden

\section{Frage 7}

Was ist das Material der Wahl zur Fissuren- und Grübchenversiegelung?
A ein Glas-lonomer-Zement
B ein niedrigvisköser methacrylatbasierter Kunststoff
$C$ ein Autopolymerisat
D ein Compomer-Material
$\mathrm{E}$ ein Composite

\section{- Weitere Fragen auf der folgenden Seite...}




\section{Punkte sammeln auf CME.thieme.de}

\section{Fortsetzung...}

\section{Frage 8}

Was ist die Hauptursache eines partiellen oder vollständigen Retentionsverlustes von Fissurenversiegelungen?
A Patient mit Behinderung
B noncomplianter Patient
C unvollständig appliziertes Versieglermaterial
D grazil appliziertes Versieglermaterial
E Materialüberschuss

\section{Frage 9}

Wie sollten partiell oder vollständig verloren gegangene Fissurenversiegelungen behandelt werden?

A Entfernung des Versieglerrestes

B regelmäßiger Recall

C klinische Beobachtung

D Reparatur bzw. Erneuerung

E keine Behandlung

\section{Frage 10}

In welchem Zeitfenster sollte das Monitoring von Fissurenversiegelungen durchgeführt werden?
A jährlich
B alle 6 bis 12 Monate
C halbjährlich
D vierteljährlich
E nicht erforderlich 